\title{
Practical Consideration on Power Line Filter Design and Implementation
}

\author{
Daria Nemashkalo*, Niek Moonen*, Frank Leferink*† \\ ${ }^{*}$ University of Twente, Enschede, the Netherlands \\ ${ }^{\dagger}$ THALES Nederland B.V., Hengelo, the Netherlands \\ dasha.nemashkalo@utwente.nl
}

\begin{abstract}
Electromagnetic interference filters have been studied extensively and proven to be a complex subject, with no single easy solution or rule of thumb. Typical filtering solutions for differential mode disturbances originating from a switched mode power supply are investigated in this paper. A simple T-shape filter model is used to derive an insertion loss equation that is dependent on source and load impedance, which allows to predict filter performance in more realistic settings compared to typical $50 / 50 \Omega$ measurement setups. It concurs with observations made in a case study that changing filter orientation could increase filter performance.
\end{abstract}

\section{INTRODUCTION}

In many cases newly developed modern equipment are not able to comply with the electromagnetic compatibility (EMC) regulations without implementing a large amount of filtering. They often have a switched mode power supply (SMPS) in their power distribution stage, as these are highly efficient. However, its efficiency comes with a price, which is an increase in electromagnetic interference (EMI) due to nonlinear behavior.

Frequently, commercial of the shelf (COTS) SMPS which are tested according to the generic standards are used for the professional applications where the EMC requirements starts at $10 \mathrm{kHz}$. In order to comply with these requirements an external filters should be used. Externally applied solutions can be categorized into passive and active filtering techniques. However, as the case study in [1] of the COTS SMPS that causes extremely high differential mode (DM) emission only at $100 \mathrm{kHz}$ shows, applying full COTS filter is not an optimal choice and may cost more in terms of finances, as well as space and weight. The future plans of the industrial partner towards this case is to connect more than 40 SMPS in parallel. Consequently, the resulted EMI level will be much higher than the measured previously, which will require more effective filtering options. Thus, comparison between a full COTS filter and a line inductor, a line to line capacitor, or a combination of the two was made. It was shown that in a specific orientation of the LC combination the COTS power line filter (PLF) was outperformed. Moreover, filters are often designed for common mode (CM) and/or DM, however EMI emission measured using a line impedance stabilisation

This project has received funding from the European Union's Horizon 2020 research and innovation programme under the Marie Skłodowska-Curie grant agreement No 812391 . network (LISN), and thus evaluating normal mode (NM). Studies like [2], emphasize often on mathematical solutions for complex filtering architectures that will predict or estimate performance over an as wide as possible frequency range. Similarly, a trend can be seen in the rise of awareness of mutual coupling effects becoming more dominant in degrading the performance of filters consisting of passive components [3], [4], and thus geometry and placement being of utmost importance [5]. A systematic approach in EMI filter design can be found in [6], where the measurements have shown a SMPS that exceeds the limit lines in nearly the entire frequency range of the CISPR 22 class B limit. Similarly in [1] a practical approach to three phase filter design was proposed. The procedure can be summarized in:

1) Determine the CM and DM suppression requirements

2) Select the topology regarding the noise source and load impedance

3) Select filter components

In practical situations it is often not the case that the excessive, i.e. above the limit lines, emissions are relatively wide band nor do they require relatively high attenuation levels, or even that they comprise of CM and DM interference simultaneously. In other words, in case of day to day engineering practises, the filters should be optimized towards disturbance specific cases instead of state-of-the-art and high performance which the authors of [6] correctly noted, and in [1] has been applied in a case study. In this paper a pragmatic view on design and choices of filters or its components will be given. To substantiate the reasoning behind choices made or advice given, a simplistic filter model will be presented and applied to the case presented in [1]. The presented model focuses on the effect of source and load impedance mismatching scenarios, showing the effect of implementation orientation of asymmetrical designed filters. The model can also be used to asses the effectiveness of the most common and elementary components of an EMI filter, practical designs and choices are emphasized in an engineering context. The filters under consideration will be passive in nature consisting only of inductors and capacitors. Based on the presented model there are 5 possible classical (equivalent) filter configurations: series inductor, shunt capacitor, or combination in 'LC', 'CL' or even ' $\mathrm{T}$ ' configuration. The practical issues are important to show, as even though many studies have been performed on EMI 
filter design, theoretically well designed filters can still fail during the integration phase [7]. The reason for performance degradation of the filter might not always easy to find, as the filter was measured in a test setup that verified its insertion loss (IL) according to for instance CISPR 17 [8]. In which case, the filter is often tested in a $50 \Omega$ source and load impedance situation, although the annex recommends $0.1 \Omega / 100 \Omega$ (and vice versa) source and load impedance testing for power line filters. Nevertheless most datasheets comprise only of data obtained in the idealistic $50 \Omega$ scenario. Next to this, it is often $\mathrm{DM}$ and/or CM performance that is shown, while in most EMI testing scenarios the emission is measured in NM.

In Sec. II a generic T-shaped filter model is presented based on complex impedances, from which an equation is derived that predicts IL for several components in different configuration under changing loading conditions. Sec. III continues with practical design considerations and downsides of using conventional DM EMI mitigation solutions. Some concluding remarks are given in Sec. IV.

\section{Filter MOdel}

In this section a simplified EMI filter model is presented, with which the theoretical performance of filtering components can be derived. As can be seen, the model also includes source and load impedances, to include possible deviations from the expected performance of a filter that has been or will be measured with the idealistic $50 \Omega$ termination.

In Fig. 1 the model is shown consiting of five distinct impedances, in which case the filter consist three of them in a "T" type of configuration. Inserting $Z_{i}=0$, with $i$ denoting any subscript seen in the figure, will be equivalent of placing a short at that position. Equating an impedance to infinity will be similar as to creating an "open".

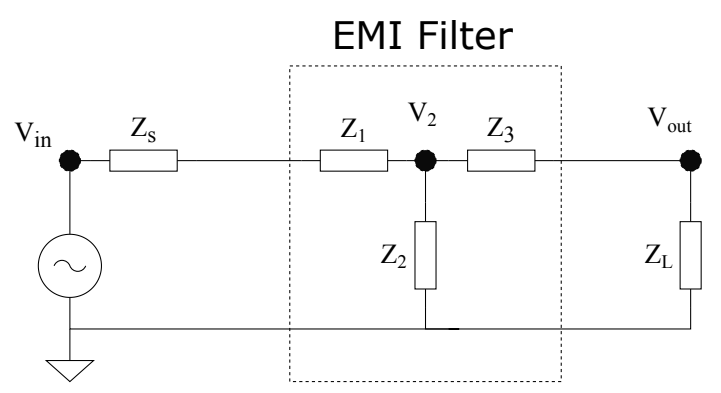

Fig. 1: Generalized equivalent circuit filter model.

To reduce the complexity of the analysis, first $Z_{1}=Z_{3}=0$ is set, so one ends up with a voltage divider of $Z_{S}$ and the parallel connection of $Z_{L}$ and $Z_{2}$. The transfer function from input to output becomes:

$$
H(\omega)=\frac{1}{1+\frac{Z_{s}}{Z_{L}}+\frac{Z_{s}}{Z_{2}}}
$$

Now simply inserting the previously neglected series impedance to the source and load impedances, i.e. $Z_{1} \neq$
$0, Z_{3} \neq 0$. It can be seen that the voltage at $V_{2}$ is:

$$
V_{2}=V_{i n} \cdot \frac{1}{1+\frac{Z_{s}+Z_{1}}{Z_{L}+Z_{3}}+\frac{Z_{s}+Z_{1}}{Z_{2}}}
$$

The voltage at $V_{2}$ is then split between $Z_{3}$ and $Z_{L}$, resulting in a total transfer function of:

$$
H(\omega)=\frac{1}{1+\frac{Z_{s}+Z_{1}}{Z_{L}+Z_{3}}+\frac{Z_{s}+Z_{1}}{Z_{2}}} \cdot \frac{Z_{L}}{Z_{L}+Z_{3}}
$$

This however does not directly show the effectiveness of a filter, and therefor the IL is calculated. The definition as given in CISPR 17 [8] Annex E is the ratio of the output voltage with and without the filter inserted, which can be mathematically displayed as:

$$
\mathrm{IL}=\frac{V_{\text {out }, \text { with }}}{V_{\text {out }, \text { without }}}=\frac{V_{\text {in }} \cdot H_{\text {in,with }}}{V_{\text {in }} \cdot H_{\text {in,without }}}
$$

Note that often the short connection between generator and receiver is skipped, as that it often assumed to be fully defined by two $50 \Omega$ impedances. Therefore it would be possible to use the generator voltage and the measured output voltage with a filter inserted directly. Following the formal definition (as in CISPR 17) the following IL was derived from the model:

$$
\begin{gathered}
Z_{e}=\frac{Z_{2} \cdot\left(Z_{3}+Z_{L}\right)}{Z_{2}+\left(Z_{3}+Z_{L}\right)} \\
\mathrm{IL}=\frac{Z_{e}}{\left(Z_{S}+Z_{1}\right)+Z_{e}} \cdot \frac{Z_{L}}{Z_{3}+Z_{L}} \cdot \frac{Z_{L}+Z_{S}}{Z_{L}}
\end{gathered}
$$

Were the last term in Eq. 6 is the inverse of the transfer function of the system without a filter. Using Eq. 6 one is able to estimate the effectiveness of filters under different loading and sourcing conditions. From Eq. 6 it can be seen that in case of a symmetrical filter, i.e. $Z_{1}=Z_{3}$, the impedance mismatch of source and load will not effect the filter's effectiveness. Impedance mismatching is shown in CISPR 17 Annex C [8] and is recommend for power line filters. It is used to predict (more accurately) in a $0.1 \Omega-100 \Omega$ system the performance under actual operating condition, and in special cases even $0.1 \Omega-1 \mathrm{M} \Omega$. The aimed frequency range of interest in these tests is $1 \mathrm{kHz}-300 \mathrm{kHz}$.

\section{A. Case Study}

In [1] a three-phase $6 \mathrm{~kW}, 380 / 480 \mathrm{~V}_{\mathrm{AC}}$ to $52 / 48 \mathrm{~V}_{\mathrm{DC}}$ converter was described which produced dominantly DM noise at $100 \mathrm{kHz}$. Fig. 3 shows NM voltage measured with a lowcost digitizer in time-domain from the output of the LISN, and converted in frequency-domain by fast Fourier transform (FFT). In the measurement setup a comparison was made between a COTS filter and the insertion of a capacitor of approx. $40 \mu \mathrm{F}$ and an inductor of approx. $1.5 \mathrm{mH}$, between and into the lines respectively. Even with an internal input filter, this converter does not comply with AECTP 500 NCE02-1 [9]. In Tab. I one can see that without knowing the source and load impedance approximately nor having datasheet information available about filter performance in impedance mismatch conditions, one might accidentally choose the least optimal 


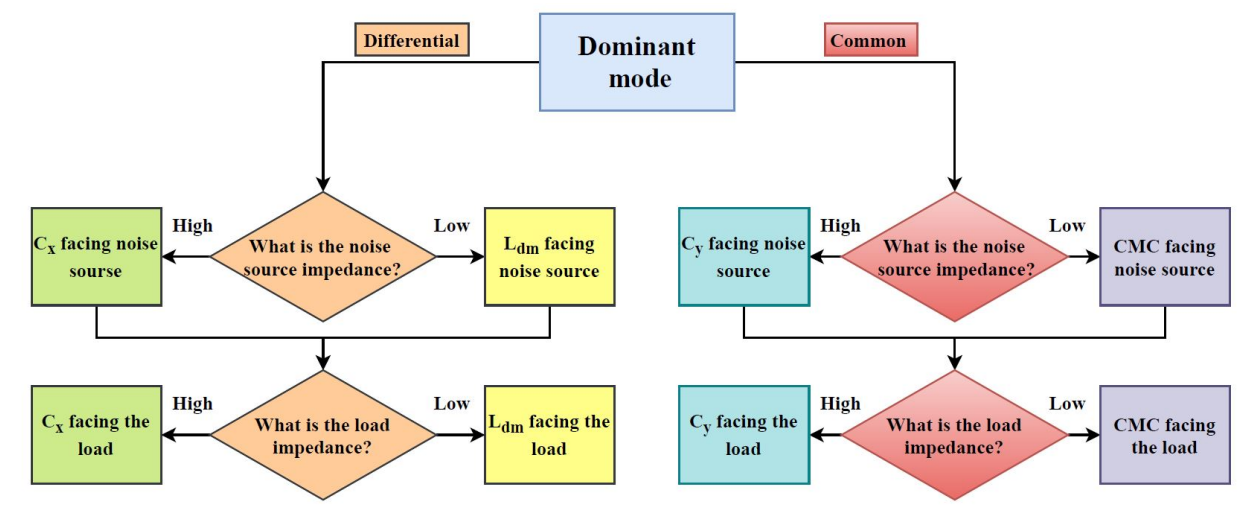

Fig. 2: Flow chart for choosing what EMI mitigation technique to apply, decisions are based on dominate mode and source and load impedances.

TABLE I: Predicted IL values in different source and load impedance conditions at $100 \mathrm{kHz}$ in comparison with the case study measurement results from [1]. The impedance notation convention is $Z_{s} / Z_{L}$.

\begin{tabular}{l|c|c|c||r}
$\mathrm{IL}$ & $50 / 50 \Omega$ & $0.1 / 100 \Omega$ & $100 / 0.1 \Omega$ & Case Study \\
\hline \hline $\mathrm{C}(40 \mu \mathrm{F})$ & $-56 \mathrm{~dB}$ & $-11 \mathrm{~dB}$ & $-11 \mathrm{~dB}$ & $-10 \mathrm{~dB}$ \\
\hline $\mathrm{L}(1.5 \mathrm{mH})$ & $-20 \mathrm{~dB}$ & $-20 \mathrm{~dB}$ & $-20 \mathrm{~dB}$ & $-15 \mathrm{~dB}$ \\
\hline $\mathrm{LC}$ & $-81 \mathrm{~dB}$ & $-87 \mathrm{~dB}$ & $-31 \mathrm{~dB}$ & $-31 \mathrm{~dB}$ \\
\hline $\mathrm{CL}$ & $-81 \mathrm{~dB}$ & $-31 \mathrm{~dB}$ & $-87 \mathrm{~dB}$ & $-55 \mathrm{~dB}$ \\
\hline LCL & $-107 \mathrm{~dB}$ & $-107 \mathrm{~dB}$ & $-107 \mathrm{~dB}$ & \\
\hline COTS & & & & $-46 \mathrm{~dB}$ \\
\hline
\end{tabular}

mitigation solution. The table, together with Eq. 6 allows for an estimation of the equivalent source impedance of the SMPS. From Fig. 4 follows that the equivalent impedance at $100 \mathrm{kHz}$ is very low and $0.1 \Omega$ would more accurately represent the source impedance than $50 \Omega$.

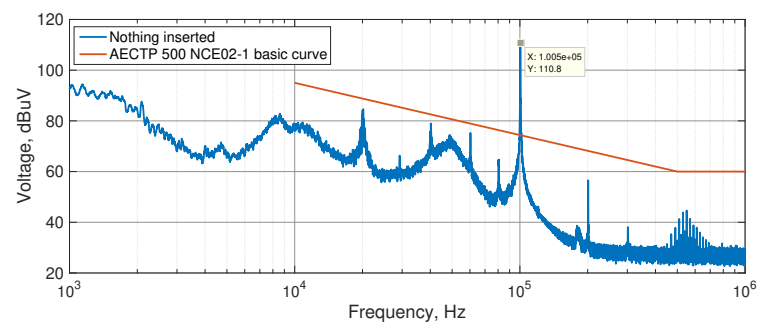

Fig. 3: The measured SMPS NM voltage

Now the IL has been derived from the model, and the influence of varying source and load impedances on the performance of the EMI mitigation technique, in the following section a novel DM EMI technique is discussed.

\section{Practical Filter Design Considerations}

Previous section has shown several DM solutions for EMI mitigation, with its basic building blocks being a set of $C_{x}$ capacitors and a large line inductor. It was already shown that their performance is heavily dependent on the source and load

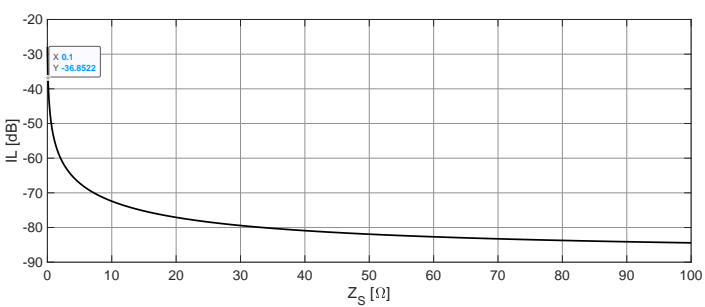

Fig. 4: IL dependency of source impedance with a fixed $50 \Omega$ load impedance, due to the LISN.

impedance. One could make a decision for either mitigation solution based solely on performance, neglecting possible size, weight, durability, life-time expectancy and cost constraints. In this section, the advantages and disadvantages with respect to the practical design will be considered and discussed.

\section{A. Differential Mode Capacitors $\left(C_{x}\right)$}

A $C_{x}$ capacitor can be inserted between phases (DM) or the phase and neutral to filter any high frequency disturbance, and preventing it to flow into the direction of for instance the mains. It will also work vice versa, as it will filter any high frequency EMI emanating from the mains, that would have potentially harmed or degraded the performance of the load. These capacitors are relatively cheap, lightweight and easy to install. However, the biggest downside is that it will also conduct the mains alternating current (AC) frequency, be it $50 \mathrm{~Hz}, 60 \mathrm{~Hz}$ or even $400 \mathrm{~Hz}$ in the form of reactive current. This introduces a serious issue in life time, due to rapid aging. Similar situations can be seen in [10], [11], where often the aging of direct current (DC) link capacitors are evaluated, it is however (amongst other factors) the alternating current flow that contributes to the performance degradation of the capacitors. In case of $C_{x}$ capacitors, the value can be nearly freely chosen. There is no safety issue in the amount of EMI being diverted to either side of the capacitor, which in case of capacitors connected to earth would provide a safety hazard $\left(C_{y}\right)$. Typical values range between a couple of $\mathrm{nF}$ and several $\mathrm{mF}$. However, there is an optimum in selecting the value, 
as increased values actually result in larger sized capacitors. As was shown in [12], [13], the high frequency performance of capacitors is dependent on geometrical dimensions, and often modeled as a parasitical series inductor (equivalent series inductor (ESL)) together with an equivalent series resistor (ESR). In general both increase with increased size, therefore an increase in the chosen capacitance value will not always be the best solution for optimized filtering.

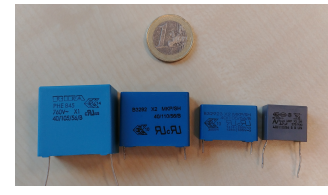

(a)

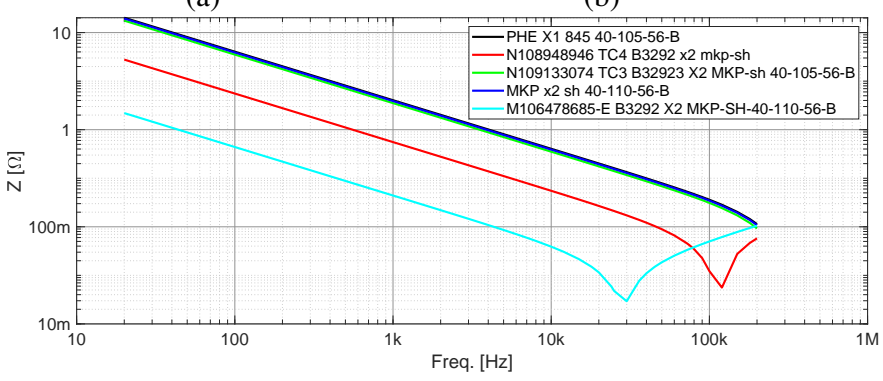

(c) Measured impedance of the shown capacitors. Legend order is from left to right.

Fig. 5: Several $C_{x}$ capacitors of different values: $0.39 \mu \mathrm{F}$, $2.8 \mu \mathrm{F}, 0.45 \mu \mathrm{F}, 0.41 \mu \mathrm{F}, 40 \mu \mathrm{F}$ from left to right. The weight of each is less than approx. $50 \mathrm{~g}$, while $\mathrm{WxLxH}$ range between $5.7 \times 3.4 \times 4.9 \mathrm{~cm}$ and $2.0 \times 1.0 \times 1.5 \mathrm{~cm}$.

\section{B. Differential Mode Inductors}

As can be seen from Tab. I, line inductors can be used as a stable and predictable EMI mitigation technique. However their implementation is often avoided. They are mostly bulky, as they require to be rated for the full loading currents inside the system. This is a big disadvantage, as they should actually not interfere with or suppress anything of these "useful" currents. The mere size and weight are key problems for being implemented in for instance automotive or aerospace, where volume and mass counts. The weight can be mostly attributed to the core material being used. Often iron/steel cores are used in these type of inductors due to a relatively high permeability (compared to air) and high saturation. However these are intertwined and thus, trying to reduce its size by increasing permeability often leads to issues with saturation. Next to this, high inductance values will introduce voltage transients as a result from switching of currents which can be seen for the voltage and current relation of an inductor.

The inductor's functionality is identical to the $C_{x}$ capacitor, i.e. to filter out the high frequency disturbance and thus act as a low pass filter. The physical mechanism is quite different though, and so are the parasitics restricting/limiting its performance. The functional behavior of the line inductor is either reflecting or absorbing the EMI propagating over the line, while the capacitor is actually diverting the disturbance and returning it to its origin. Reflection occurs due to impedance mismatch between the noise source and "load" impedance. In which case the inductor is actually contributing to the perceived "load" impedance. As opposed to the capacitor, the parasitical behavior will actually bypass the inductor at high frequencies. This is also a well known phenomena and often modeled with an equivalent parallel capacitor (EPC) and equivalent parallel resistor (EPR), as for instance shown in [14]-[16]. Its parasitic behavior is, similarly to the capacitor, mainly governed by geometrical characteristics, for instance winding structure, winding density, and input to output distance.

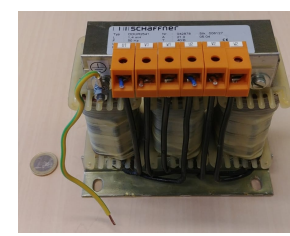

(a)

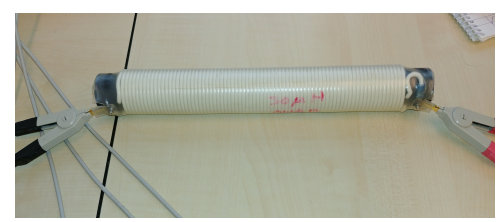

(b)

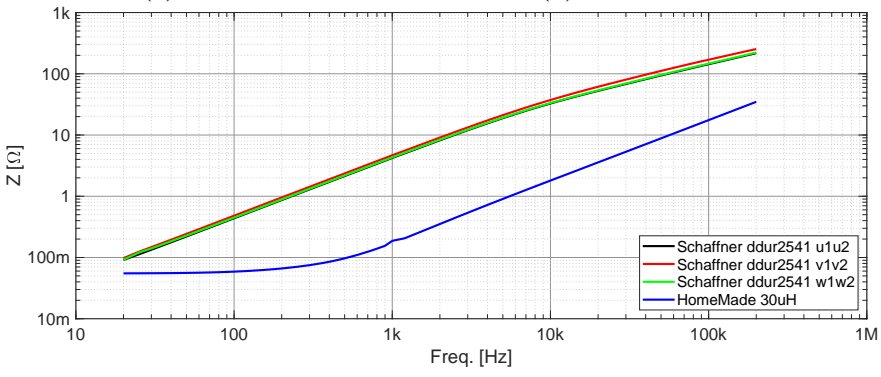

(c) Measured impedance for above shown 3-phase inductor for each phase separately.

Fig. 6: 6a) Typical 3-phase line inductor of $1.4 \mathrm{mH}$ of approx. $7.5 \mathrm{~kg}$ and $\mathrm{WxLxH} 15 \times 10 \times 14 \mathrm{~cm}$. 6b) A homemade aircore inductor of $30 \mu \mathrm{H}$ of approx. $300 \mathrm{~g}$ and DxL being approx. $40 \mathrm{~mm} \times 28 \mathrm{~cm}$.

It has been shown that both typical solutions, the $C_{x}$ and line inductor, do not provide an ideal method of filtering. Be it due to leakage currents degrading the lifetime of the capacitor or due to the size and weight of the inductor. Combining this practical discussion with the model presented in Fig. 1, it can be seen that the physical characteristic of the series connected impedance is directly related to the power flowing through it and thus inherently a costly and bulky option. However by trading off capacitor size and inductor values, one is able to reduce the cost, size and weight of the EMI mitigation solution.

\section{Trade-offs applied to case study}

Being able to trade-off capacitor and inductor values, sizes, weight and eventually costs might not be as straightforward as expected. Knowledge of source and load impedances need to used to effectively optimize the filter's performance, and thus to chose wisely during trade-offs. During the case study 
described in Sec. II-A, an inductor of $1.5 \mathrm{mH}$ needed to be combined with a capacitor of $40 \mu \mathrm{F}$ and placed in the correct orientation to achieve the minimal suppression of $40 \mathrm{~dB}$. This reduced the total cost of the solution, however its weight was similar to the COTS filter and depending on implementation, the space required was very similar. Most significant contributor of total weight is the inductor with approximately $7.5 \mathrm{~kg}$. By losing its core material the inductor will become lighter, however it will also be less inductive. Using Fig. 7 one can determine the minimum inductance needed. This was done for several capacitor values, as these have (individually) almost no significant impact on the total size, weight and cost of the mitigation solution. To emphasize the importance of taking into account impedance mismatching between source and load, not only the IL is shown for a $50 / 50 \Omega$ situation (Fig. 7a) but also for $0.1 / 50 \Omega$ (Fig. 7b). The latter represent the situation of conducted EMI emission measurements. It shows that in the traditional filter evaluation, one requires high inductance values before it becoming an effective suppression tool. This can be seen by the dropoff/"cutoff" value of approx. $100 \mu \mathrm{H}$, while in case of a low source impedance the effectiveness is dependent on the capacitor used and ranging between $100 \mathrm{nH}$ and $10 \mu \mathrm{H}$. However, in that case the capacitor becomes less effective, as was seen in the case study and expected from the analytical analysis.

From Fig. $7 \mathrm{~b}$ it follows that the homemade inductor from Fig. $6 \mathrm{~b}$, of $30 \mu \mathrm{H}$, requires a capacitor of at least $5 \mathrm{nF}$ to achieve a theoretical suppression of $40 \mathrm{~dB}$. Even when using the $40 \mathrm{nF}$ will still be large improvement in weight reduction, as almost $6.5 \mathrm{~kg}$. Note the volume is similar, due to need for three homemade inductors.

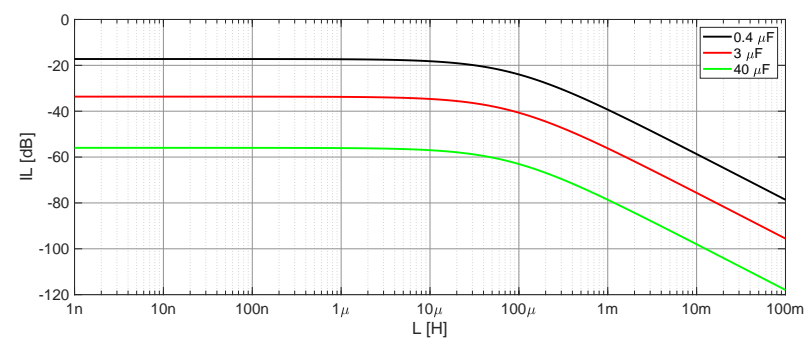

(a) Assumed $Z_{S}=50 \Omega$, and $Z_{L}=50 \Omega$ (due to the LISN).

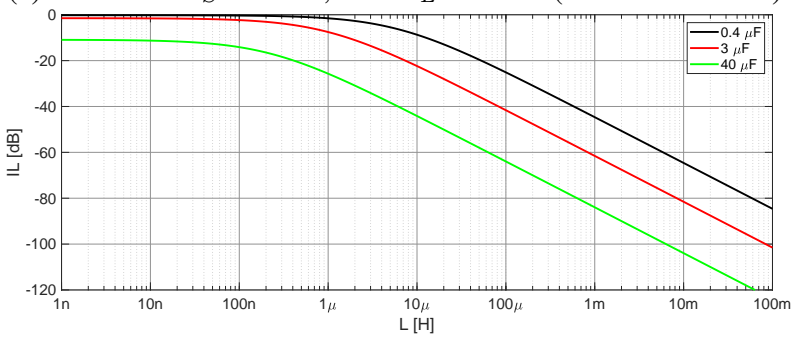

(b) Assumed $Z_{S}=0.1 \Omega$, and $Z_{L}=50 \Omega$ (due to the LISN).

Fig. 7: Insertion Loss calculated in an LC filter for different inductor and capacitor values, at $100 \mathrm{kHz}$ and optimal orientation.

\section{CONCLUSION}

A fundamental study has been done to find the best solution for the DM filters needed in terms of achieving the high performance while sustaining optimal filter size, weight, and costs, which is very significant for the industry. Issues related to COTS filters, that are not specified below $150 \mathrm{kHz}$, not specified for NM, impedance mismatch and not specified for issued like capacitors aging due to reactive currents, are addressed. This paper has provided an generic equation for determining the insertion loss of a filter under impedance mismatch conditions, i.e. different source and load impedances. The derivation was based on a three element Tshape impedance model, hence there is an inherent limitation to the number of filter configurations that it can be used for. It is used in this paper to predict how the conventional elements, capacitor and inductor in several configurations, would perform in different measurement scenarios. The practical limitations/considerations of using DM inductors have been discussed, ranging from transients due to off-switching to core material being heavy and saturate-able. In case of capacitors, the limitations are leakage currents reducing lifetime and being non-effective in low-impedance situations. It has been shown by understanding the to be mitigated EMI, selective trade-offs can be made to reduce filter size, weight and costs, while maintaining performance.

\section{REFERENCES}

[1] D. Nemashkalo, N. Moonen, and F. Leferink, "Multi-channel timedomain EMI evaluation of dominant mode interference for optimized filter design in three-phase systems," vol. 3, no. 1, pp. 6-9.

[2] D. C. Micic, "An Analytical Approach for DC-Link DM Filter Design for PM AC Motor Drives," in Joint IEEE International Symposium on Electromagnetic Compatibility \& Asia-Pacific Symposium on Electromagnetic Compatibility. Singapore: IEEE, 2018, pp. 1240-1247.

[3] G. Asmanis, D. Stepins, A. Asmanis, and L. Ribickis, "Mutual Couplings between EMI Filter Components," in IEEE International Symposium on Electromagnetic Compatibility. Dresden: IEEE, 2015, pp. 908-913.

[4] G. Asmanis and D. Stepins, "Effects of Parasitic Parameters on Three Phase EMI Filters," in Topical Problems in the Field of Electrical and Power Engineering, 2012, pp. 93 - 102.

[5] N. Moonen, F. Buesink, and F. Leferink, "Optimizing capacitor placement in EMI-filter using back annotation of 3D field coupling parameters in circuit models," in 2016 International Symposium on Electromagnetic Compatibility - EMC EUROPE. Wroclaw: IEEE, sep 2016, pp. 576580.

[6] V. Tarateeraseth, K. Y. See, F. G. Canavero, and R. W. Y. Chang, "Systematic electromagnetic interference filter design based on information from in-circuit impedance measurements," IEEE Transactions on Electromagnetic Compatibility, vol. 52, no. 3, pp. 588-598, 2010.

[7] N. Moonen, F. Buesink, and F. Leferink, "Unexpected poor performance of presumed high-quality power line filter, and how it improved," in 2015 IEEE International Symposium on Electromagnetic Compatibility (EMC). Dresden: IEEE, aug 2015, pp. 382-385.

[8] CISPR 17, "Methods of measurement of the suppression characteristics of passive EMC filtering devices," 2011.

[9] NATO International Staff - Defence Investment Division Allied Environmental Conditions and Tests Publication AECTP500 (edition 4), "Electromagnetic Environmental Effects Test And Verification-Equipment and sub-system tests," 2011.

[10] M. A. Vogelsberger, T. Wiesinger, and H. Ertl, "Life-cycle monitoring and voltage-managing unit for DC-link electrolytic capacitors in PWM converters," IEEE Transactions on Power Electronics, vol. 26, no. 2, pp. 493-503, 2011. 
[11] J. Hannonen, J. Honkanen, J. P. Ström, T. Kärkkäinen, S. Räisänen, and P. Silventoinen, "Capacitor Aging Detection in a DC-DC Converter Output Stage," IEEE Transactions on Industry Applications, vol. 52, no. 4, pp. 3224-3233, 2016.

[12] G. Asmanis, D. Stepins, A. Asmanis, and L. Ribickis, "Capacitors mutual inductance modeling and reduction," in International Symposium on Electromagnetic Compatibility, EMC Europe. Gothenburg, Sweden: IEEE, 2014, pp. 1176-1181. [Online]. Available: http://ieeexplore.ieee.org/lpdocs/epic03/wrapper.htm?arnumber=693108

[13] N. Moonen, F. Buesink, and F. Leferink, "Enhanced circuit simulation using mutual coupling parameters obtained via 3D field extraction," in 2016 Asia-Pacific International Symposium on Electromagnetic Compatibility (APEMC). Shenzhen, China: IEEE, may 2016, pp. 181-183.
[14] S. Wang, F. C. Lee, and W. G. Odendaal, "Characterization and parasitic extraction of EMI filters using scattering parameters," IEEE Transactions on Power Electronics, vol. 20, no. 2, pp. 502-510, 2005.

[15] A. Asmanis, G. and Ribickis, L. and Stepins, D. and Asmanis, "Differential Mode Pi-type EMI filter modeling using CST MWS," in Power and Electrical Engineering of Riga Technical University (RTUCON), 2015 56th International Scientific Conference on, 2015, pp. 1-5.

[16] N. Moonen, A. Roc'h, and F. Leferink, "Influence of mutual coupling on parasitic capacitance in common mode chokes," in 2018 IEEE International Symposium on Electromagnetic Compatibility and 2018 IEEE Asia-Pacific Symposium on Electromagnetic Compatibility (EMC/APEMC). Singapore: IEEE, may 2018, pp. 1305-1309. 